\title{
Towards Patient-Specific Finite-Element Simulation of MitralClip Procedure
}

\author{
T. Mansi ${ }^{1}$, I. Voigt ${ }^{1,2}$, E. Assoumou Mengue ${ }^{1}$, R. Ionasec ${ }^{1}$, B. Georgescu ${ }^{1}$, \\ T. Noack ${ }^{3}$, J. Seeburger ${ }^{3}$, and D. Comaniciu ${ }^{1}$
}

1 Siemens Corporate Research, Image Analytics and Informatics, Princeton, NJ, USA

2 Friedrich-Alexander-University, Pattern Recognition Lab, Erlangen, Germany

3 Department of Cardiac Surgery, Heart Centre Leipzig, Leipzig, Germany

\begin{abstract}
MitralClip is a novel minimally invasive procedure to treat mitral valve (MV) regurgitation. It consists in clipping the mitral leaflets together to close the regurgitant hole. A careful preoperative planning is necessary to select respondent patients and to determine the clipping sites. Although preliminary indications criteria are established, they lack prediction power with respect to complications and effectiveness of the therapy in specific patients. We propose an integrated framework for personalized simulation of MV function and apply it to simulate MitralClip procedure. A patient-specific dynamic model of the MV apparatus is computed automatically from $4 \mathrm{D}$ TEE images. A biomechanical model of the MV, constrained by the observed motion of the mitral annulus and papillary muscles, is employed to simulate valve closure and MitralClip intervention. The proposed integrated framework enables, for the first time, to quantitatively evaluate an MV finite-element model in-vivo, on eleven patients, and to predict the outcome of MitralClip intervention in one of these patients. The simulations are compared to ground truth and to postoperative images, resulting in promising accuracy (average point-to-mesh distance: $1.47 \pm 0.24 \mathrm{~mm}$ ). Our framework may constitute a tool for MV therapy planning and patient management.
\end{abstract}

\section{Introduction}

The mitral valve (MV), between the left atrium and the left ventricle, prevents the blood from coming back to the left atrium during systole. Incorrect MV closure appears in many cardiac diseases and often requires surgery. The edgeto-edge technique, which consists in suturing the two mitral leaflets at the regurgitant hole, has demonstrated good clinical outcomes in patients with severe mitral insufficiency due to leaflet prolapse or calcified annulus [7. Nowadays, this procedure can be performed percutaneously by clipping the leaflets using a MitralClip catheter [3]. Nonetheless, a careful preoperative planning is necessary to select respondent patients and to determine the clipping sites. Although preliminary indications criteria are established, they lack prediction power with respect to complications and effectiveness of the therapy in specific patients. It is not uncommon to perform several trials during the intervention and, in some

G. Fichtinger, A. Martel, and T. Peters (Eds.): MICCAI 2011, Part I, LNCS 6891, pp. 452-459 2011.

(C) Springer-Verlag Berlin Heidelberg 2011 
cases, decide to place two clips $(\approx 30 \%$ of the patients $[3])$ or even to abort the procedure due to complications $(\approx 10 \%$ of the patients $[3])$. Therefore, there is a need for an efficient and predictive framework that can assist the surgeon in planning the MitralClip procedure and guide him during the intervention.

Driven by the growing prevalence of MV diseases, researchers are developing computational models of MV biomechanics to simulate its function. Several constitutive laws have been proposed, from simple isotropic linear elasticity to more complex anisotropic non-linear hyper-elasticity [911/13. Fluid-structure interaction models have also been investigated [2. Yet, most of these models have been developed on synthetic or ex-vivo anatomies [4. Patient-specific anatomies and boundary conditions are starting to be used but tedious manual delineations are still required, with no [12] or partial automation [113. Recently, a patientspecific simulation of MV annuloplasty has been presented [12] but the results were not confronted to postoperative data. At the same time, automatic algorithms are being developed to delineate the MV in medical images. In [5], the authors provided a fast and accurate method based on machine learning to detect the MV on 3D+t transesophageal echocardiogram (TEE) or CT images. All the elements are starting to be available for patient-specific MV simulations.

We thus propose in this paper to combine data-driven modeling of the MV with a biomechanical model of the valve apparatus to simulate MV closure in patients and test therapies, in particular the MitralClip procedure. As described in Sec. 2, we use machine-learning techniques to automatically detect the complete MV apparatus in sequences of 3D TEE images. A biomechanical model of the MV is then employed to simulate valve closure on the patient-specific anatomies and boundary conditions. As model generation is automatic and integrated, we could quantitatively evaluate the simulations with respect to the observed valve motion in eleven subjects (Sec. 3). In one of these patients, post- MitralClip images were available. The intervention was simulated and compared with the real outcome, showing promising prediction power.

\section{Methods}

Starting from 4D TEE images, we automatically detect the MV apparatus in all time frames using machine-learning algorithms and generate a patient-specific anatomical model of the open MV (Sec. 2.1). We then apply a biomechanical model to simulate valve closure (Sec. (2.2) and valve clipping (Sec. 2.3).

\subsection{Anatomical Model of the Mitral Valve Apparatus}

The anatomical model of the MV apparatus that is detected in the images comprises (Fig. 1): the mitral annulus, the anterior and posterior leaflets (AL and PL resp.), the anterior and posterior papillary heads and the chordae. To capture a broad spectrum of morphological variations, the model is parameterized by three coarse-to-fine components: $i$ ) Three transforms $B$ for global location, orientation and scale over the cardiac cycle; ii) The trajectories of ten anatomical landmarks 


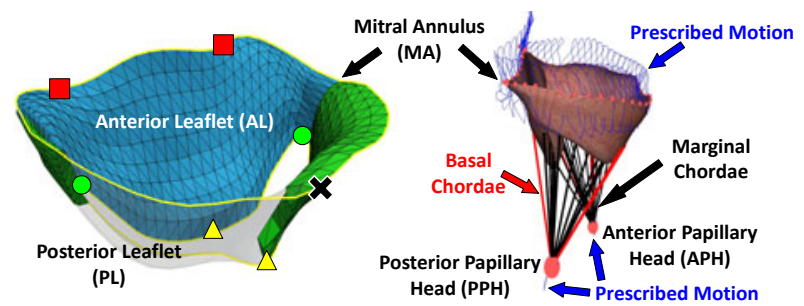

Fig. 1. Anatomical model of the MV and subvalvular apparatus, with 2 trigones (squares), 1 posterior annulus mid-point (cross), 2 commissures (circles) and 2 leaflet tips (triangles). The tetrahedral mesh (left) is tagged for regional tissue properties.

$L(B)=\left(\mathbf{l}_{1} \ldots \mathbf{l}_{10}\right) \in \mathbb{R}^{3 \times 10}$ (see Fig. 1); and iii) Two triangulated surface meshes $S_{L A}(B, L)$ and $S_{L V}(B, L)$ to represent the left atrial (LA) and left ventricular $(\mathrm{LV})$ leaflet surfaces respectively. The vertices of each surface are constrained by the landmarks, resulting in an anatomically consistent parameterization that ensures intra- and inter-patient point correspondence.

$B, L(B)$ and $S_{L A}(L, B)$ are personalized from the images using a hierarchical discriminative learning algorithm [5. The probability $p(B, L, S \mid I)$ knowing the image data $I$ is incrementally modeled within the Marginal Space Learning (MSL) framework, based on the Probabilistic Boosting Tree (PBT) 14. Due to the poor image quality, it is still difficult to estimate the thickness of the leaflets automatically and reliably at every time frame of the cardiac sequence. We therefore obtain the LV leaflet surface $S_{L V}$ by artificially extruding $S_{L A}$ in the direction of the surface normals, towards the $\mathrm{LV}$, by $2 \mathrm{~mm}$, the average leaflet thickness measured in our patients (see Sec. 3).

Finally, we generate a tetrahedral volume mesh of the MV (Fig. 1). For regional personalization, the tetrahedra are tagged according to the leaflet they belong to thanks to the anatomically consistent surface parameterization. 30 marginal chordae are evenly attached between papillary heads and leaflet free edges as well as four sets of two basal chordae, two sets for each leaflet. Insertion points, identical for every patient according to the point correspondence inherited from the anatomical model, are determined by visual inspection of the images, when visible, or like in previous studies [413] otherwise.

\subsection{Biomechanical Model of Mitral Valve Apparatus}

Valve closure is simulated by solving the dynamic system $\mathrm{M} \ddot{\mathbf{U}}+\mathrm{C} \dot{\mathbf{U}}+\mathrm{KU}=$ $\mathbf{F}_{c}+\mathbf{F}_{p} . \mathbf{U}$ is the displacement vector of the free vertices of the MV mesh, $\dot{\mathbf{U}}$ their velocity and $\ddot{\mathbf{U}}$ their acceleration. $\mathrm{M}$ is the diagonal mass matrix (leaflet mass density $\rho=1.04 \mathrm{~g} / \mathrm{mL}), \mathrm{K}$ is the stiffness matrix of the internal elastic forces and $\mathrm{C}$ is a Rayleigh damping matrix with coefficient 0.1 for both $\mathrm{M}$ and $\mathrm{K} . \mathbf{F}_{c}$ and $\mathbf{F}_{p}$ are the forces developed by the chordae and heart pressure respectively.

Leaflets are near-incompressible, anisotropic, non-linear elastic [10]. In this study we are not directly interested in leaflet stresses but we rather seek to 
predict how well they close to assess possible residual regurgitant holes after MitralClip intervention. Hence, we approximate the MV properties by a linear isotropic elastic model [4, which optimizes the computational efficiency for fast simulations in the operative room. Near-incompressibility is achieved with a Poisson ratio $\nu$ of 0.488 . The AL being stiffer than the PL, two different Young moduli are used, $\mathrm{E}_{A L}=2.08 \mathrm{MPa}$ and $\mathrm{E}_{P L}=1.88 \mathrm{MPa}$ respectively 11. In this study we considered the cross-fiber stiffness (perpendicular to the mitral annulus), lower than the fiber stiffness, to capture the radial deformations.

Chordae are modeled by piecewise tensile springs between papillary heads and insertion points (Fig. 1), $\mathbf{F}_{c, i}=-k_{c, i}\left(\epsilon_{c, i}\right) \times\left(L_{i}-L_{i, 0}\right), i \in\{$ marginal, basal $\}$. $L_{i}$ is the current elongation and $L_{i, 0}$ is the rest length defined as the distance between the papillary heads and the insertion points measured at middiastole. The stiffness $k_{c, i}\left(\epsilon_{c, i}\right)$ depends on the strain $\epsilon_{c, i}=\left(L_{i}-L_{i, 0}\right) / L_{i, 0}$ to model the non-linear response of the chordae. At compression, $\epsilon<0, k_{c, i}=$ $0 \mathrm{~g} / \mathrm{mm}$ (free compression). At low tension $\epsilon<2.5 \%$, chordae exhibit low stressstrain behavior (Young moduli $E_{c, \text { basal }}=66 \mathrm{~g} / \mathrm{mm}^{2}, E_{c, \text { marginal }}=312 \mathrm{~g} / \mathrm{mm}^{2}$ ), which then increases dramatically and almost linearly $\left(E_{c, \text { basal }}=2120 \mathrm{~g} / \mathrm{mm}^{2}\right.$, $E_{c, \text { marginal }}=3406 \mathrm{~g} / \mathrm{mm}^{2}$ ) [6]. Spring stiffnesses $k_{c, i}$ are calculated from the chordae Young moduli by $k_{c, i}=A_{0, i} E_{c, i} / L_{0, i} . A_{0, i}$ is the chordae cross-section at rest $\left(A_{0, \text { basal }}=2.05 \mathrm{~mm}^{2}, A_{0, \text { marginal }}=0.40 \mathrm{~mm}^{2}\right)$.

Pressures being not available, we apply a generic profile that increases from $0 \mathrm{mmHg}$ to $120 \mathrm{mmHg}$. The motion of the papillary heads, modeled as spatial points, and of the mitral annulus is prescribed from the automatic detection. This contribution is of fundamental importance as valve closure highly depends on the papillary positions and the shape of the annulus during systole 9]. As mitral clip modifies the leaflets morphology only, it is reasonable to assume that the acute motion of the annulus and papillaries stays unchanged (it depends mostly on the ventricular mechanics). Self-collisions and frictions are handled using collision springs.

The models are implemented in $\mathrm{SOFA}^{1}$, a real-time soft-tissue intervention platform. The dynamic system is solved using co-rotational tetrahedral finite elements to cope with large deformations and rotations 8 . The simulation time is personalized and scaled such that the simulated MV closure is $10 \times$ longer than what is observed in the images (from $70 \mathrm{~ms}$ to $150 \mathrm{~ms}$ ) to handle the strong and discontinuous contact forces. An implicit Euler solver is used to update mesh positions. We finally stress that the pipeline is completely integrated and automatic, although the user can manually adjust the models if necessary.

\subsection{MitralClip Simulation}

Virtual mitral clipping is performed interactively on the preoperative anatomical model as illustrated in Fig. 2. Stiff springs $\left(k_{c l i p}=1000 \mathrm{~g} / \mathrm{mm}\right)$ are created between the two leaflets to simulate the clip. Tissue properties are like in Sec. 2.2

1 http://www.sofa-framework.org 

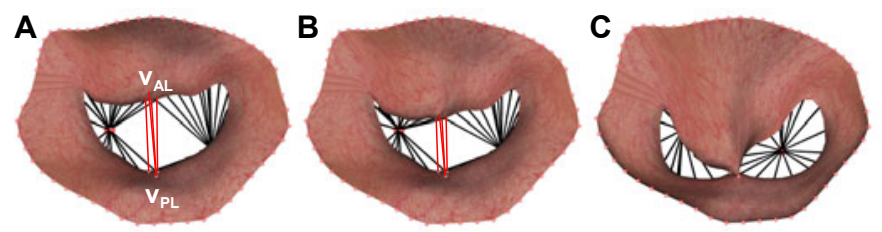

Fig. 2. Virtual MitralClip procedure. (A) The user picks two vertices $\mathbf{v}_{\mathbf{A L}}$ and $\mathbf{v}_{\mathbf{P L}}$ on each leaflet to create springs (red lines) that will (B) progressively bring the leaflets close to each other and $(\mathbf{C})$ stitch them. The procedure is done interactively.

\section{Experiments and Results}

Validation of the MV Anatomical Model. Automatic detection of the MV and its subvalvular apparatus was quantitatively validated on 200 4D TEE images from 120 patients with various diseases (MV prolapse, calcified mitral annulus, stenosis, ventricular dysfunction, ....). Images were acquired with different capture range and image resolutions. Three-fold cross-validation against manual delineation yielded a point-to-mesh error of $2.75 \pm 0.86 \mathrm{~mm}$ (detection speed: $4.8 \mathrm{~s}$ per 3D volume, Intel Core2Duo, $2.66 \mathrm{GHz}$ quad core, $2 \mathrm{~GB}$ RAM). As shown in Fig. 3, the model was able to faithfully track the MV even during valve closure.

Evaluation of MV Closure Simulation. Our simulation framework was evaluated on eleven randomly selected patients from three hospitals with various degrees of MV regurgitation or MV stenosis. The complete MV apparatus was detected, under expert guidance, on clinical 4D TEE images (image resolution: $0.75-1.58 \mathrm{~mm}$ isotropic, $8-23$ time frames). Papillary heads could be detected reliably as they were visible on all images. The anatomical model at end-diastole, when the valve is fully open, was used to simulate MV closure. Tetrahedral meshes showed no skewed cells. The detected motion of the mitral annulus and papillary heads were used as boundary conditions. Because the biomechanical parameters of the leaflets and chordae are difficult to identify in clinical data, we decided to use the nominal parameters reported in Sec. 2 for every patient. This enabled us to evaluate the generalization of such an approach for future clinical use. Table 1 reports the average point-to-mesh distances from the simulations to the

3D Volume

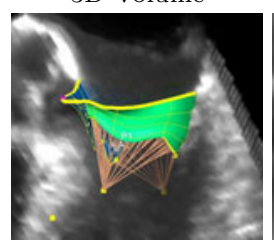

2D Views during Valve Closure
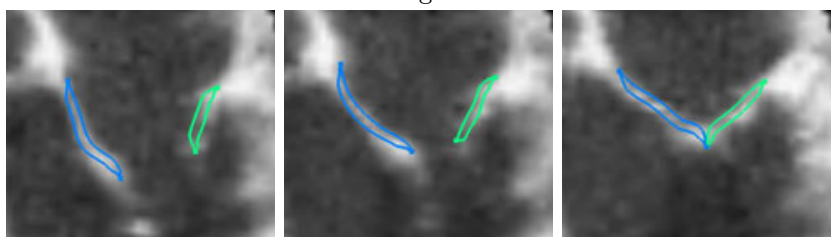

Fig. 3. Automatic detection of the mitral valve on a time sequence of 3D TEE. As one can see, the detected model faithfully tracks the moving valve over time. 
Table 1. Point-to-mesh distance between simulated and detected closed configuration of the MV valve. In average, the error $(1.47 \pm 0.24 \mathrm{~mm})$ was of the same order of magnitude as that of the automatic detection $(2.75 \pm 0.86 \mathrm{~mm})$.

\begin{tabular}{|cc|cc|cc|}
\hline Patient Point-to-Mesh Error & Patient & Point-to-Mesh Error & Patient & Point-to-Mesh Error \\
\hline 01 & $1.33 \pm 0.77 \mathrm{~mm}$ & 05 & $1.56 \pm 1.24 \mathrm{~mm}$ & 09 & $2.00 \pm 1.62 \mathrm{~mm}$ \\
02 & $1.16 \pm 0.68 \mathrm{~mm}$ & 06 & $1.36 \pm 0.82 \mathrm{~mm}$ & 10 & $1.53 \pm 1.42 \mathrm{~mm}$ \\
03 & $1.56 \pm 1.14 \mathrm{~mm}$ & 07 & $1.36 \pm 0.89 \mathrm{~mm}$ & 11 & $1.25 \pm 0.80 \mathrm{~mm}$ \\
04 & $1.31 \pm 0.81 \mathrm{~mm}$ & 08 & $1.76 \pm 1.40 \mathrm{~mm}$ & \multicolumn{2}{|c}{} \\
\end{tabular}

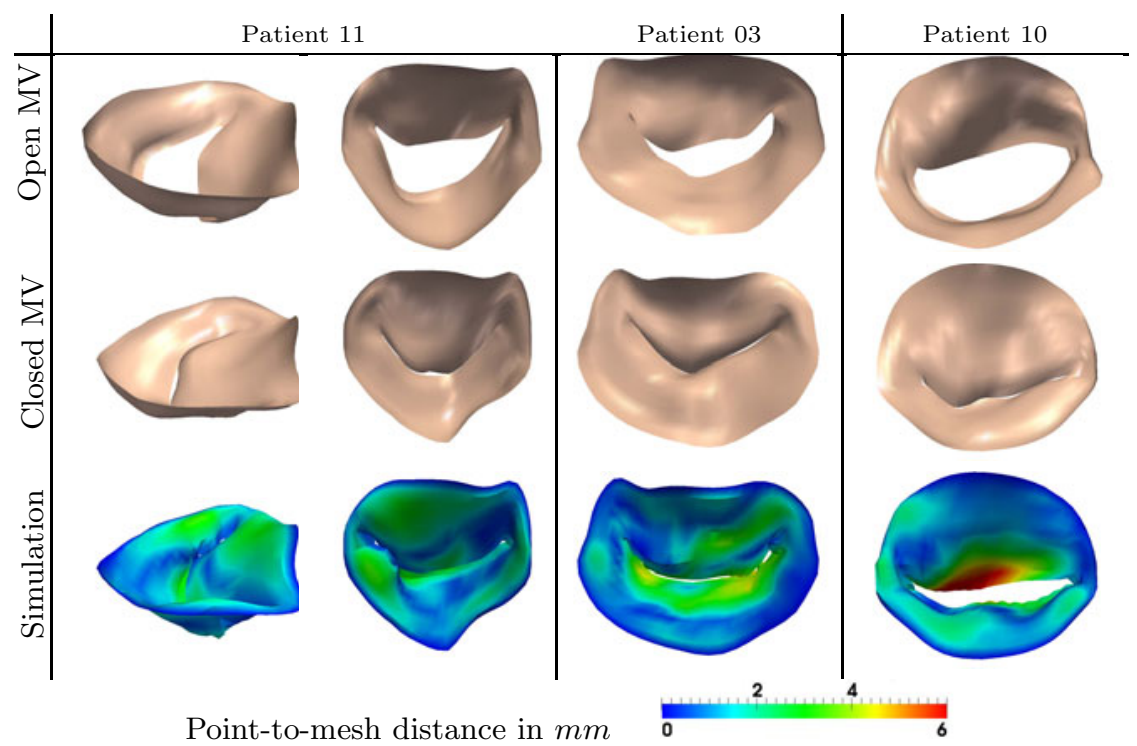

Fig. 4. Simulated MV closure in three patients compared to observed MV shape. In seven out of eleven cases, the biomechanical model managed to simulate valve closure, as in patient 03 and 11 . In four patients, some chordae were too short to achieve a correct closure, as in patient 10. See text for details.

anatomical models detected on the first frame when the MV is closed. The average error was $1.47 \pm 0.24 \mathrm{~mm}$, which is of the same order of magnitude of the automated detection. The biomechanical model correctly reproduced valve closure in seven patients (63\%) despite the simplified leaflet model and the generic parameters (Fig. 4, left and mid panels). On the other four patients, the valve did not coapt correctly (Fig. 4, right panel). Nonetheless, correct coaptation could be simulated by adjusting the rest length of selected chordae, which confirms their importance in MV function 9. Simulation speed was $\approx 4$ frames per second $(f p s)$. Total simulation time was $\approx 20 s$ with a time-step $\Delta t=10 \mathrm{~ms}$ (Intel Core2Duo, 2.66GHz dual core, 4GB RAM). Non-reported simulations with $\Delta t=\{1 \mathrm{~ms}, 0.1 \mathrm{~ms}\}$ yielded very similar results, confirming the temporal convergence of the simulation. The entire process, from the TEE images to the simulation, took about one minute. 


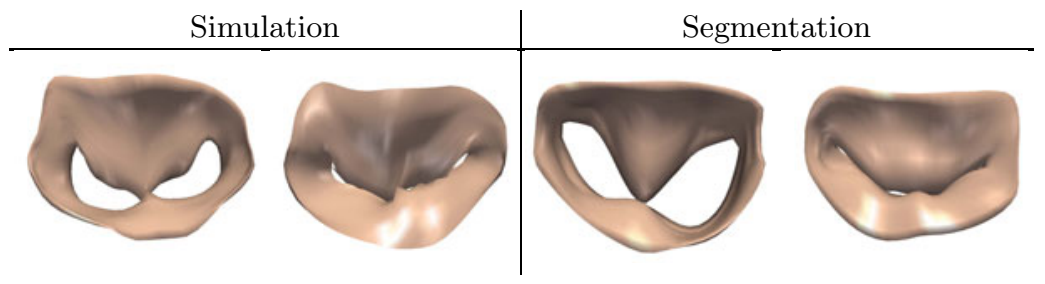

Fig. 5. To assess the ability of the model to predict MitralClip outcomes, the intervention was simulated on the preoperative anatomy of a patient (Fig. 4 patient 1), with preoperative boundary conditions (left panel). The result was qualitatively consistent with the true postoperative outcome (right panel).

Simulation of Mitral Clip. We tested the ability of our model to predict the acute outcome of MitralClip intervention in one patient (patient 3) for whom intra-operative images just after clip release were available. Since the simulation of valve closure on the preoperative anatomy was successful, we could reproduce the intervention on the preoperative anatomy and simulate the subsequent valve closure (Fig. 2, frame-rate: $8 \mathrm{fps}$ ) using the preoperative biomechanical parameters and boundary conditions (mitral annulus and papillary heads motion). Results were qualitatively similar to the real surgical outcome (Fig. 河).

\section{Discussion and Future Works}

We proposed in this paper an integrated framework for the personalized simulation of MV closure and MitralClip procedure. Our approach, fully automatic, enables easy and computationally efficient simulations. We could evaluate a patient-specific biomechanical model of the MV in eleven patients. Results demonstrated that despite the simplifications of our model and the generic parameters, good prediction power can be achieved by using patient-specific anatomies and mitral annulus and papillary heads motion. We also simulated MitralClip intervention in one patient, resulting in promising predictions compared to postoperative data. In some patients, valve closure could be achieved only by adjusting chordae biomechanical parameters (rest length and stiffness). It is therefore important to model chordae correctly for accurate and predictive simulations. As a first step, we proposed to estimate chordae rest length from the mid-diastole images, when they are at rest. One could use inverse problem methods to further personalize them, along with MV stiffness. Yet, detecting the insertion points remains a technical challenge. The proposed framework can be easily extended to other imaging modalities like TTE or CT, wherever the MV is visible. Future works include the development of non-linear anisotropic model of MV [13, simulation of valve opening to assess the effects of MitralClip on the diastolic function, anatomical model improvement and validation on larger cohorts. To the best of our knowledge, it is the first time that a biomechanical model of the MV is evaluated against in-vivo clinical data in a predictive manner. As illustrated by our MitralClip simulation, our framework could open, once validated, new perspectives to test MV therapies in-silico to optimize treatment outcome. 
Acknowledgement. The authors warmly thank E. Pernod for his help with SOFA.

\section{References}

1. Burlina, P., Sprouse, C., DeMenthon, D., Jorstad, A., Juang, R., Contijoch, F., Abraham, T., Yuh, D., McVeigh, E.: Patient-specific modeling and analysis of the mitral valve using 3d-tee. In: Proc. IPCAI, pp. 135-146 (2010)

2. Einstein, D., Del Pin, F., Jiao, X., Kuprat, A., Carson, J., Kunzelman, K., Cochran, R., Guccione, J., Ratcliffe, M.: Fluid-structure interactions of the mitral valve and left heart: Comprehensive strategies, past, present and future. International Journal for Numerical Methods in Biomedical Engineering 26(3-4), 348-380 (2010)

3. Feldman, T., Kar, S., Rinaldi, M., Fail, P., Hermiller, J., Smalling, R., Whitlow, P., Gray, W., Low, R., Herrmann, H., et al.: Percutaneous mitral repair with the mitraclip system: Safety and midterm durability in the initial everest (endovascular valve edge-to-edge repair study) cohort. JACC 54(8), 686-694 (2009)

4. Hammer, P., Vasilyev, N., Perrin, D., Del Nido, P., Howe, R.: Fast image-based model of mitral valve closure for surgical planning. In: Metaxas, D., Axel, L., Fichtinger, G., Székely, G. (eds.) MICCAI 2008, Part II. LNCS, vol. 5242, pp. 15-26. Springer, Heidelberg (2008)

5. Ionasec, R., Voigt, I., Georgescu, B., Wang, Y., Houle, H., Vega-Higuera, F., Navab, N., Comaniciu, D.: Patient-specific modeling and quantification of the aortic and mitral valves from 4D cardiac CT and TEE. IEEE TMI 29(9), 1636-1651 (2010)

6. Kunzelman, K., Cochran, K.: Mechanical properties of basal and marginal mitral valve chordae tendineae. ASAIO Journal 36(3), M405 (1990)

7. Maisano, F., Torracca, L., Oppizzi, M., Stefano, P., d'Addario, G., La Canna, G., Zogno, M., Alfieri, O.: The edge-to-edge technique: a simplified method to correct mitral insufficiency. European Journal of Cardio-thoracic Surgery 13(3), 240 (1998)

8. Nesme, M., Payan, Y., Faure, F.: Efficient, physically plausible finite elements. In: Eurographics (short papers), pp. 77-80 (2005)

9. Prot, V., Haaverstad, R., Skallerud, B.: Finite element analysis of the mitral apparatus: annulus shape effect and chordal force distribution. Biomechanics and Modeling in Mechanobiology 8(1), 43-55 (2009)

10. Sacks, M., Yoganathan, A.: Heart valve function: a biomechanical perspective. Phil. Trans. R. Soc. B 362(1484), 1369 (2007)

11. Schievano, S., Kunzelman, K., Nicosia, M., Cochran, R., Einstein, D., Khambadkone, S., Bonhoeffer, P.: Percutaneous mitral valve dilatation: Single balloon versus double balloon. a finite element study. J. Heart Valve Disease 18, 28-34 (2009)

12. Stevanella, M., Maffessanti, F., Conti, C., Votta, E., Arnoldi, A., Lombardi, M., Parodi, O., Caiani, E., Redaelli, A.: Mitral valve patient-specific finite element modeling from cardiac MRI: Application to an annuloplasty procedure. In: Cardiovascular Engineering and Technology, pp. 1-11 (2011)

13. Votta, E., Caiani, E., Veronesi, F., Soncini, M., Montevecchi, F., Redaelli, A.: Mitral valve finite-element modelling from ultrasound data: a pilot study for a new approach to understand mitral function and clinical scenarios. Phil. Trans. R. Soc. A 366(1879), 3411 (1879)

14. Zheng, Y., Barbu, A., Georgescu, B., Scheuering, M., Comaniciu, D.: Four-chamber heart modeling and automatic segmentation for 3D cardiac CT volumes using marginal space learning and steerable features. IEEE TMI 27, 1668-1681 (2008) 